\title{
Violent mergers of nearly equal-mass white dwarf as progenitors of subluminous Type la supernovae
}

\author{
R. Pakmor ${ }^{1,2}$, S. Hachinger ${ }^{1}$, F. K. Röpke ${ }^{1,3}$, and W. Hillebrandt ${ }^{1}$ \\ 1 Max-Planck-Institut für Astrophysik, Karl-Schwarzschild-Str. 1, 85741 Garching, Germany \\ e-mail: rpakmor@mpa-garching.mpg.de \\ 2 Heidelberger Institut für Theoretische Studien, Schloss-Wolfsbrunnenweg 35, 69118 Heidelberg, Germany \\ 3 Institut für Theoretische Physik und Astrophysik, Universität Würzburg, Am Hubland, 97074 Würzburg, Germany
}

Received 27 August 2010 / Accepted 3 February 2011

\begin{abstract}
Context. The origin of subluminous Type Ia supernovae (SNe Ia) has long eluded any explanation, because all Chandrasekhar-mass models have severe problems reproducing them. Recently, it has been proposed that violent mergers of two white dwarfs of $0.9 M_{\odot}$ could lead to subluminous SNe Ia events that resemble 1991bg-like SNe Ia.

Aims. Here we investigate whether this scenario still works for mergers of two white dwarfs with a mass ratio below one. We aim to determine the range of mass ratios for which a detonation still forms during the merger, as only those events will lead to an SN Ia. This range is an important ingredient for population synthesis and one decisive point for judging the viability of the scenario. In addition, we perform a resolution study of one of the models. Finally we discuss the connection between violent white dwarf mergers with a primary mass of $0.9 M_{\odot}$ and 1991 bg-like SNe Ia.

Methods. The latest version of the smoothed particle hydrodynamics code Gadget 3 was used to evolve binary systems with different mass ratios until they merge. We analyzed the result and looked for hot spots in which detonations can form.

Results. We show that mergers of two white dwarfs with a primary white dwarf mass of $\approx 0.9 M_{\odot}$ and a mass ratio more than about 0.8 robustly reach the conditions we require for igniting a detonation and thus produce thermonuclear explosions during the merger itself. We also find that, while our simulations do not yet completely resolve the hot spots, increasing the resolution leads to conditions that are even more likely to ignite detonations. Additionally, we compare the abundance structure of the ejecta of the thermonuclear explosion of two merged white dwarfs with data inferred from observations of a 1991bg-like SN Ia (SN 2005bl). The abundance distributions of intermediate mass and iron group elements in velocity space agree qualitatively, and our model reproduces the lack of material at high velocities inferred from the observations.

Conclusions. The violent merger scenario constitutes a robust possibility for two merging white dwarfs to produce a thermonuclear explosion. Mergers with a primary white dwarf mass of $\approx 0.9 M_{\odot}$ are very promising candidates for explaining subluminous SNe Ia. This would imply that subluminous SNe Ia form a distinct class of objects, which are not produced in the standard single white dwarf scenario for SNe Ia, but instead arise from a different progenitor channel and explosion mechanism.
\end{abstract}

Key words. supernovae: general - hydrodynamics - binaries: close

\section{Introduction}

Type Ia supernovae are among the brightest objects in the Universe. Their apparent homogeneity makes them one of the most important probes for cosmic expansion. This application relies on an empirical luminosity calibration (e.g. Phillips 1993). Only recently has this calibration been justified by theoretical studies, once models were able to reproduce it in terms of the underlying physics (Kasen et al. 2009; Mazzali et al. 2001). The standard SNe Ia (Branch et al. 1993) used as cosmological distance indicators make up about 70\% ( $\mathrm{Li}$ et al. 2010) of the observed $\mathrm{SNe}$ Ia. There is, however, a number of subclasses of $\mathrm{SNe}$ Ia consisting of very dim (e.g. SN 1991bg, Leibundgut et al. 1993), normal, bright (e.g. SN 1991T, Filippenko et al. 1992) and very bright supernovae (e.g. SN 2007if, Scalzo et al. 2010). Until today, neither the progenitor scenario nor the explosion mechanism of SNe Ia have been completely understood. In particular, very dim and very bright objects are difficult to explain. There is only general consensus that all SNe Ia are thermonuclear explosions of white dwarfs (WDs) in binary systems. The nature of the companion star and the details of the explosion mechanism, however, are still being debated.

Current progenitor models distinguish between the single degenerate and the double degenerate scenario. The former (proposed by Whelan \& Iben 1973) assumes a main sequence or giant star, i.e. a non-degenerate star, to be the companion of the exploding white dwarf. It feeds the white dwarf via Roche-lobe overflow or by winds until the white dwarf approaches the Chandrasekhar mass and explodes. In contrast, the double degenerate scenario (Iben \& Tutukov 1984; Webbink 1984) assumes a binary system of two carbon-oxygen white dwarfs. Despite being favored by stellar population synthesis studies (e.g. Ruiter et al. 2009), this scenario has received little attention (see, e.g. Hillebrandt \& Niemeyer 2000). Benz et al. (1990) found that in a binary system of a $1.2 M_{\odot}$ and a $0.9 M_{\odot}$ white dwarf the less massive white dwarf is disrupted and accreted onto the more massive white dwarf. This happens within a few orbits after mass transfer between the stars has started. It has also been shown (e.g. Nomoto \& Iben 1985; Saio \& Nomoto 1998) that the most likely result of such a merger is an inward propagating deflagration flame that converts the white dwarf into an O-Ne 
white dwarf. This white dwarf then undergoes accretion-induced collapse rather than thermonuclear explosion (Nomoto \& Kondo 1991). Recent high-resolution simulations of white dwarf mergers with careful treatment of the initial conditions (D'Souza et al. 2006; Motl et al. 2007; Dan et al. 2009), however, show indications that the immediate disruption of the less massive white dwarf may be avoided. Instead, they find stable mass transfer for more than 50 orbits. When the less massive secondary WD is disrupted after these orbits, the accretion onto the primary is slower, so carbon shell ignition may be avoided (Yoon et al. 2007).

In the Chandrasekhar-mass scenario, a white dwarf is thought to ignite near the center. At first the flame propagates subsonically as a deflagration, but burns only a fraction of the star. The energy release expands the star, and in a later stage of the explosion, a detonation is triggered that propagates supersonically. The mechanism that leads to the formation of the detonation is still an open question. One of the most promising scenarios is a deflagration to detonation transition by turbulence (e.g. Khokhlov et al. 1997; Röpke \& Niemeyer 2007; Röpke et al. 2007a). Other proposed mechanisms are the gravitationally confined detonation (e.g. Jordan et al. 2008; Meakin et al. 2009) and pulsating reverse detonation (Bravo \& García-Senz 2006). A thermonuclear explosion may also be possible before the white dwarf reaches the Chandrasekhar-mass if the carbonoxygen white dwarf accretes helium from a non-degenerate or white dwarf companion (Fink et al. 2007, 2010; Sim et al. 2010; Guillochon et al. 2010).

A more detailed comparison of different explosion mechanisms can be found in Hillebrandt \& Niemeyer (2000). However, none of the Chandrasekhar-mass models are able to explain very $\operatorname{dim} \mathrm{SN}$ Ia with a ${ }^{56} \mathrm{Ni}$ mass of $0.2 M_{\odot}$ or less.

Recently, Pakmor et al. (2010) have proposed that these $\mathrm{SNe}$ Ia are the result of a violent merger of two equally massive white dwarfs. Once a binary system of two white dwarfs has formed, gravitational wave emission leads to continuous shrinking of its orbit. At some point, the two white dwarfs are close enough to interact directly. If the mass ratio of the two white dwarfs is close to unity, the interaction becomes very violent and the slightly less massive, hence less compact, white dwarf is destroyed. Its material plunges onto the remaining white dwarf, is compressed, and heats up. At the interface between the merging WDs, material can become hot enough for carbon to ignite. As carbon burning starts, it is heated up further. If these hot spots occur at high enough densities, they can reach the conditions that are needed to ignite a detonation.

The detonation then propagates through the merged object and burns most of the original carbon-oxygen material to heavier nuclei. As the detonation flame moves supersonically with respect to the fuel, and the timescale for the flame to cross the whole star is much shorter than the dynamical timescales involved, the system is burned nearly instantaneously at the moment the detonation is ignited. With enough energy released from nuclear burning to overcome the gravitational binding energy, the merged object explodes and finally reaches a state of homologous expansion.

In this work, we explore mergers of white dwarfs with a primary mass of $0.9 M_{\odot}$ and mass ratios between 0.8 and one. We investigate how the conditions during the merging event change with mass ratio and determine for which range of mass ratios the merger will produce a thermonuclear explosion.

The paper is structured as follows. In Sect. 2, the SPH code used in this work is summarized and the modifications are presented that are introduced to adapt the code to the problem. The initial setup is described in Sect. 3. Section 4 investigates inspiral and merger of one specific binary system and discusses the conditions that lead to the ignition of a detonation. Section 5 compares mergers with different mass ratios. In Sect. 6 we compare the outcome of a thermonuclear explosion following a violent merger with observations. Conclusions are drawn in Sect. 7.

\section{Implementation}

To simulate the inspiral of the binary system, the latest version of the smoothed particle hydrodynamics (SPH) code GADGET (Springel 2005) is used with some modifications. These are needed as GADGET was originally written for cosmological applications. The most important modifications to the code are the implementation of a stellar equation of state and a nuclear reaction network. For the equation of state $(\mathrm{EoS})$ of the electron gas, positrons and radiation we used a tabulated version of the EoS by Timmes \& Arnett (1999), in which we interpolate linearly. The ions were treated as a fully ionized ideal gas.

We implemented a 13 -isotope nuclear reaction network. It contains all $\alpha$-elements up to ${ }^{56} \mathrm{Ni}\left({ }^{4} \mathrm{He},{ }^{12} \mathrm{C},{ }^{16} \mathrm{O},{ }^{20} \mathrm{Ne},{ }^{24} \mathrm{Mg}\right.$, $\left.{ }^{28} \mathrm{Si},{ }^{32} \mathrm{~S},{ }^{36} \mathrm{Ar},{ }^{40} \mathrm{Ca},{ }^{44} \mathrm{Ti},{ }^{48} \mathrm{Cr},{ }^{52} \mathrm{Fe},{ }^{56} \mathrm{Ni}\right)$. The nuclear reaction rates were taken from the latest (2009) release of the REACLIB database (Rauscher \& Thielemann 2000). The network was solved in each timestep for all active particles whose temperatures exceed $10^{6} \mathrm{~K}$. It was solved separately from the hydrodynamical equations employing operator splitting. Typically the network requires significantly smaller timesteps than the hydrodynamical timesteps; therefore, multiple network timesteps had to be performed during one hydrodynamical timestep. The change in composition was taken into account for the EoS. The energy release (or consumption) due to changes in the composition is added to the internal energy of a particle. As a consequence of the implementation of the stellar equation of state and the nuclear network we solved the energy equation instead of the entropy equation (as in the original version of GADGET). Details and tests of these modifications will be discussed in Pakmor et al. (in prep.).

The configuration of the GADGET code has been very similar to its previous use in Pakmor et al. (2008). All particles have the same mass. The smoothing length is chosen such that each particle has 50 neighbor particles. Only the tree method is used to calculate the gravitational forces . The gravitational softening length of a particle is set equal to its smoothing length.

\section{Simulation setup}

In a first step, a one-dimensional model of a carbon-oxygen white dwarf in hydrostatic equilibrium is constructed. This is done by integrating the equation of hydrostatic equilibrium over the mass profile of a white dwarf, starting at the center with a given central density and assuming a constant temperature and nuclear composition throughout the whole star. The central density is chosen to give the desired mass of the white dwarf.

All white dwarfs are assumed to have a constant temperature of $T=5 \times 10^{5} \mathrm{~K}$ and a uniform composition of $X_{\mathrm{C}}=X_{\mathrm{O}}=0.5$. To set up the binaries we created white dwarfs of $M=0.90 M_{\odot}$, $M=0.70 M_{\odot}, M=0.76 M_{\odot}, M=0.81 M_{\odot}$, and $M=0.89 M_{\odot}$, respectively.

In a second step, each one-dimensional white dwarf model is mapped to a distribution of SPH particles resembling its onedimensional profile. Details of this procedure will be described in Pakmor et al. (in prep.). All particles of all white dwarfs represent the same mass. The particle mass is chosen such that the 

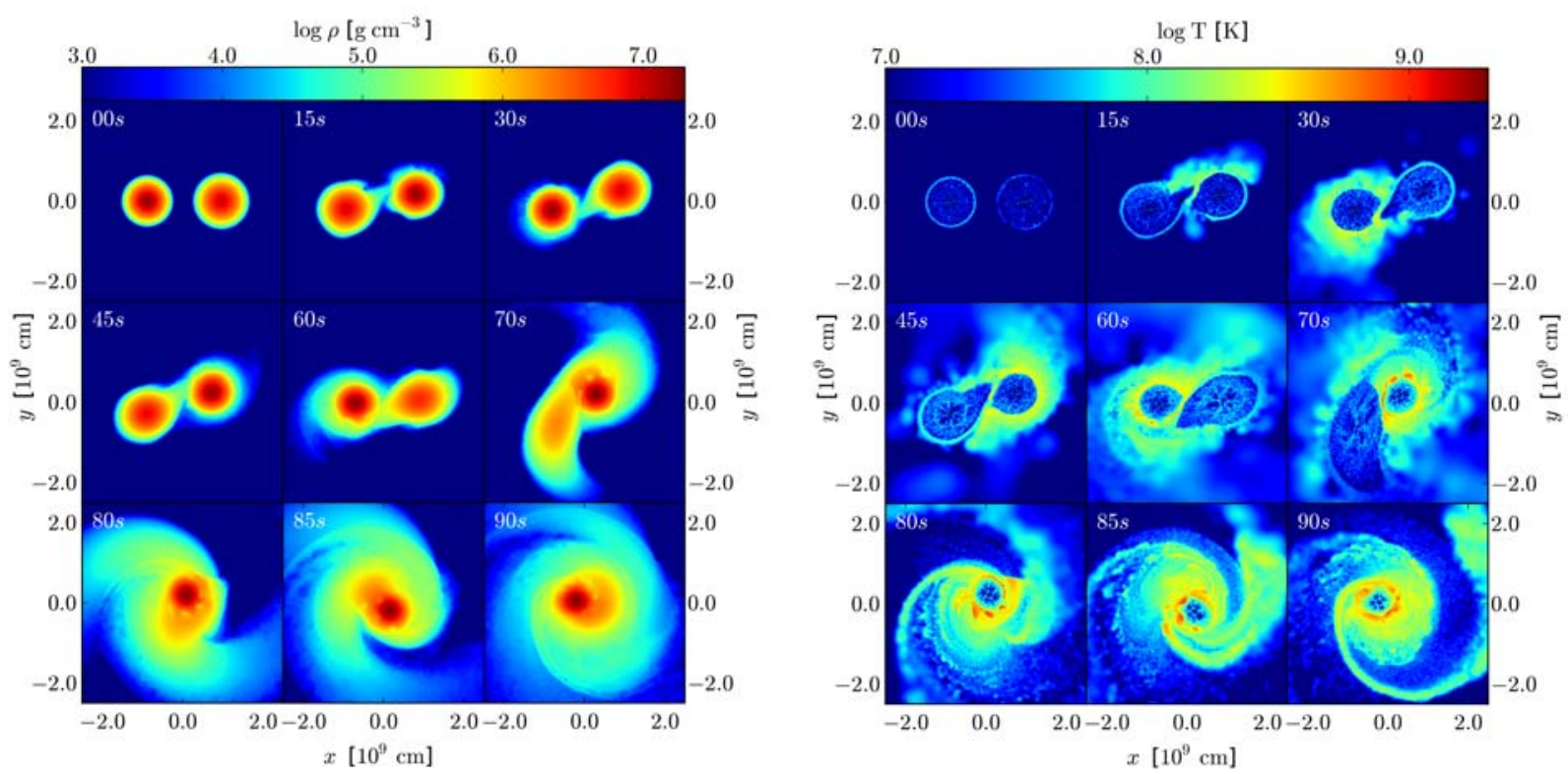

Fig. 1. Snapshots of the evolution of the binary system during the inspiral. The system contains two white dwarfs of $0.90 M_{\odot}$ and $0.81 M_{\odot}$, respectively. It was set up with an initial orbital period of 33s, rotating clockwise. Color-coded are the logarithm of the density on the left and of the temperature on the right.

most massive white dwarf $\left(M=0.90 M_{\odot}\right)$ consists of $10^{6}$ particles. Using the GADGET code, every model is finally relaxed for $100 \mathrm{~s}$ to damp out spurious numerical noise introduced by the setup.

After relaxation, the white dwarf models are used to construct binaries. Both stars in such a binary are set onto a circular orbit around their combined center of mass. All particles of each star are given the same initial velocity. The period is chosen to provide a marginally stable orbit; i.e. the system is stable for more than one complete orbit before tidal interactions lead to dynamical interactions between the white dwarfs.

This setup does not relax the binary system once the white dwarfs feel the tidal forces of their companion. Performing such an additional relaxation step may lead to more stable initial conditions and therefore a less violent merger, but to investigate this question would be computationally very demanding.

\section{Inspiral and merger of a $0.90 M_{\odot}+0.81 M_{\odot}$ white dwarf binary}

The binary evolution in the inspiral and merger phase is illustrated in Fig. 1 for the example of a merger between a $0.90 M_{\odot}$ and a $0.81 M_{\odot}$ white dwarf. Shown are snapshots of density and temperature in slices through the orbital plane $(z=0)$. The binary system rotates clockwise. It is stable for about two orbits, but both stars are slightly distorted by tidal forces. In the beginning, only the less massive white dwarf is distorted, while the more massive star stays relatively unaffected for much longer.

Mass transfer sets in a few seconds after the start of the simulation. After about two orbits ( $\approx 60 \mathrm{~s}$ ), the secondary white dwarf starts to break up, and its material is accreted rapidly onto the primary white dwarf. In this process, material on the surface of the primary white dwarf is strongly heated up, first by compression and later also by nuclear reactions to temperatures well above $10^{9} \mathrm{~K}$. This process starts with compression that subsequently leads to the ignition of carbon burning. The temperature increase beyond $1.5 \times 10^{9} \mathrm{~K}$ is due to the energy release in this nuclear reaction.

The evolution of the binary is qualitatively similar to previous studies of merging double degenerate systems (e.g. Guerrero et al. 2004; Yoon et al. 2007; Lorén-Aguilar et al. 2009). In these studies, the less massive WD is disrupted in the merger and its material builds up a hot envelope around the remaining white dwarf. Lorén-Aguilar et al. (2009) also find that when material of the secondary first hits the primary, the temperature at the interface becomes high enough to start carbon fusion. In their simulations, this leads to an rapid increase in the temperature there awing to nuclear energy release. As the temperature increases, the material becomes non-degenerate, expands and cools down, thereby quenching the nuclear reactions. In the end, only a very small amount of material is burned.

There is, however, an important difference in the interpretation. Lorén-Aguilar et al. (2009) do not consider the possibility of a detonation forming at the hotspot. In this case the detonation will be able to burn the whole white dwarf. This is a direct consequence of the detonation propagating supersonically and therefore burning all the material before it can expand.

As a result, the crucial question that decides the fate of a violent merger event is whether the merging process leads to the formation of a detonation or not. Unfortunately, this process and the conditions leading to it are still not completely understood, but are a topic of active research (e.g. Seitenzahl et al. 2009; Röpke et al. 2007b).

\subsection{Detonation ignition mechanisms}

There are basically two ways a detonation can be triggered. First, a strong enough shock may lead to direct formation of a detonation. In this case, an already existing shock wave compresses and heats the material, causing nuclear burning behind the shock. If the nuclear energy release behind the shock becomes strong enough, it may be sufficient to create a self-sustained detonation (e.g. Body 1997). Since the merger is very violent, we 


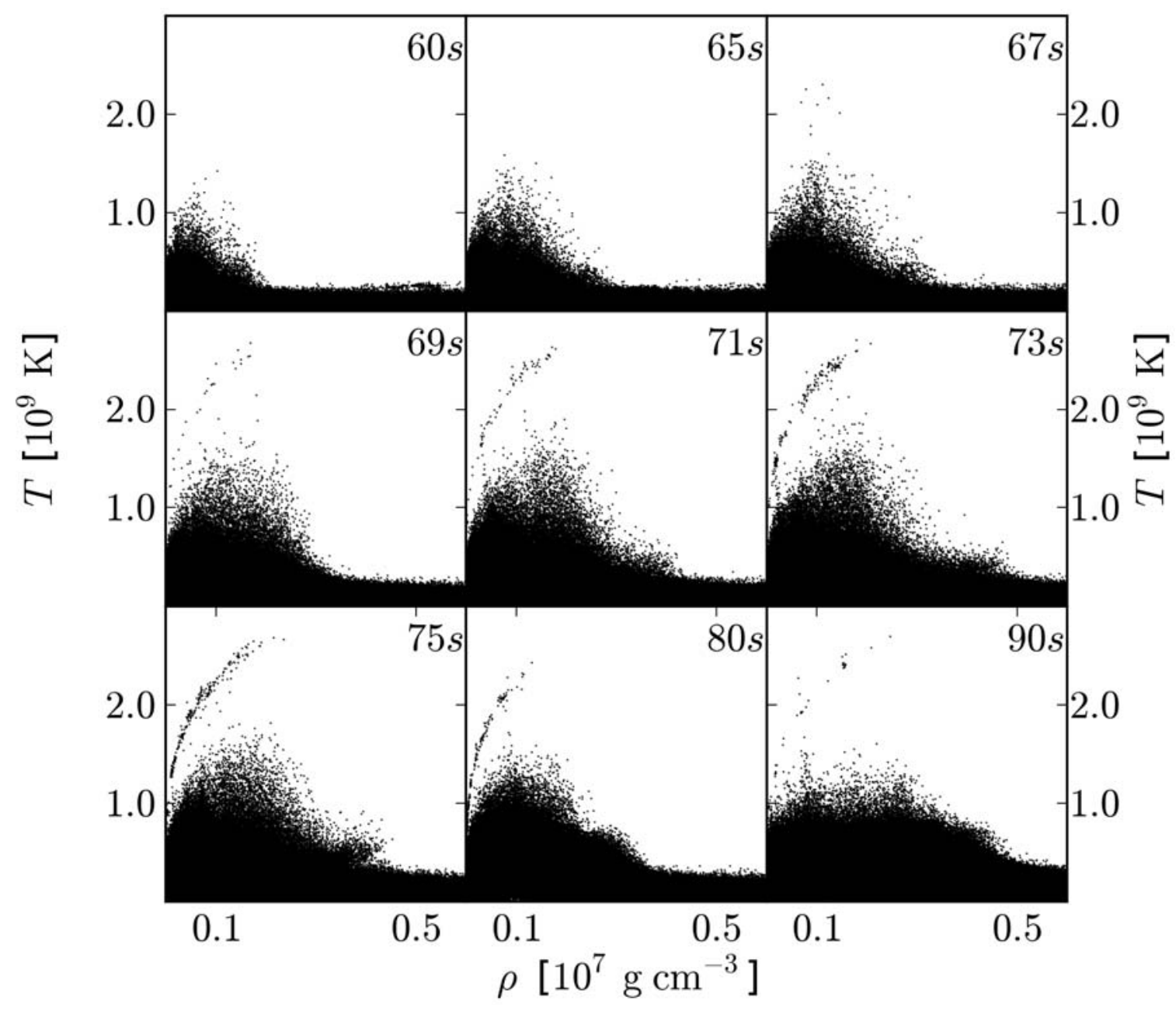

Fig. 2. Density vs. temperature scatter plot for all particles of the merger of a $0.90 M_{\odot}$ and a $0.81 M_{\odot}$ white dwarf.

naturally expect strong shocks to occur in the interaction region. Unfortunately, however, we are far from being able to resolve these shocks on small enough scales to observe the formation of a detonation. Because all SPH particles in our simulation have the same mass, the mass resolution is constant. Therefore regions with higher density are resolved better than regions with low density. Given that the interaction between the two white dwarfs occurs at comparatively low densities, their spatial resolution in the interaction region is intrinsically worse than at the center of the white dwarfs. In fact, we only barely resolve shocks in the interaction region at all. Therefore we are not able to judge from our current simulations whether a detonation can form directly from a shock. Since typical detonation flames are roughly a few meters in width and the best resolved regions in our simulations are on the order of $10^{5} \mathrm{~m}$, only future simulations with very high adaptivity may be able to resolve their formation in global simulations.

Second, it may be possible to form a detonation spontaneously without a preceding shock. In this case, as first proposed by Zel'dovich et al. (1970), nuclear burning in a preconditioned region (i.e. with a spatial temperature or composition gradient that causes an induction time gradient) creates a spontaneous ignition wave. If the preconditioning is favorable and this ignition wave has a supersonic phase velocity, it forms a shock wave that is followed by nuclear burning. But only if the release of nuclear energy behind the shock wave is sufficient to sustain the shock after it reaches the bottom of the gradient, does it become a selfsustained detonation.

Because the mechanism itself cannot be resolved in our simulations, we have to rely on studies that model the mechanism for different conditions in a microscopic region. Such studies provide an indication whether a detonation is expected to form for the physical conditions in our simulation. In a recent study, Seitenzahl et al. (2009) carried out resolved one-dimensional simulations of the gradient mechanism for different initial conditions (i.e. varying temperature, density, and steepness of the gradient). They show that the density threshold to form a detonation in carbon-oxygen material is $10^{6} \mathrm{~g} \mathrm{~cm}^{-3}$ for the most favorable conditions of geometry and gradient. At this density, a temperature above $2.8 \times 10^{9} \mathrm{~K}$ is required over a range of some meters. For higher densities, the temperatures required drop. In the following, we require a hot-spot with a density higher than $2 \times 10^{6} \mathrm{~g} \mathrm{~cm}^{-3}$ and a temperature above $2.5 \times 10^{9} \mathrm{~K}$ for concluding that a detonation forms.

\subsection{Conditions reached in the merger simulation}

Figure 2 shows density versus temperature of all particles for several snapshots from $60 \mathrm{~s}$ to $90 \mathrm{~s}$ after the start of the simulation in a scatter plot. This is the time range when the secondary white dwarf, is disrupted, its material is violently accreted onto the primary white dwarf and a hot-spot at the interface emerges. Afterwards, the temperature drops again as the hot material 


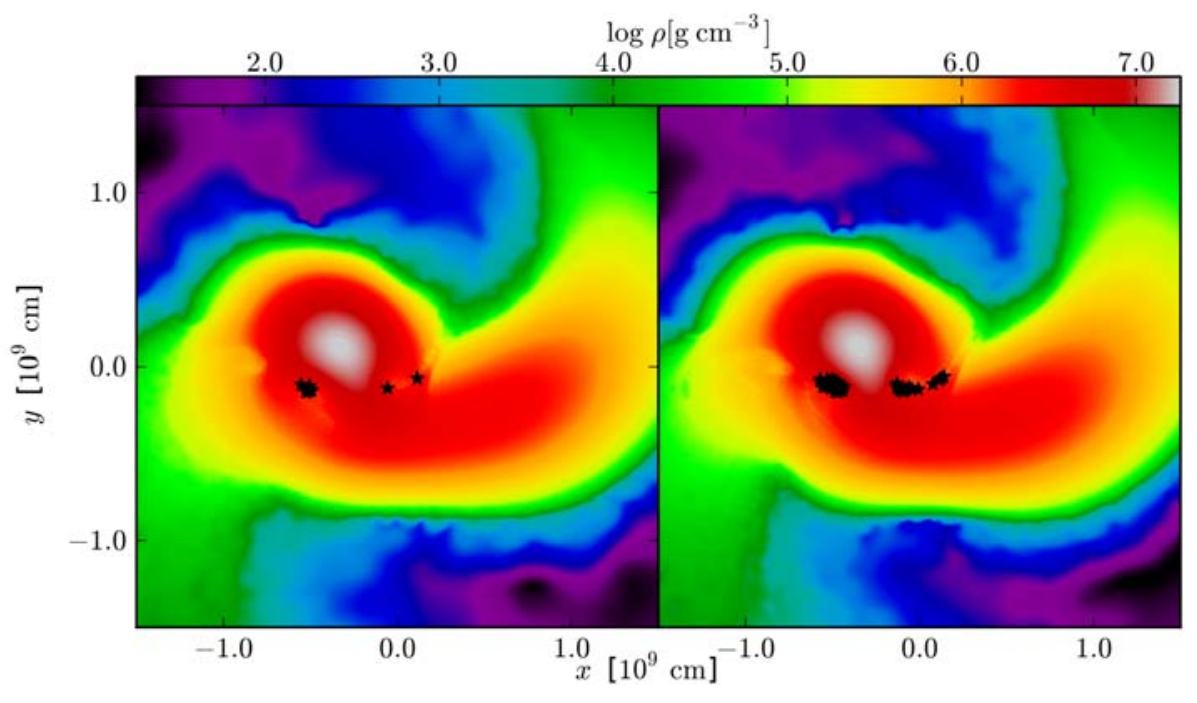

Fig. 3. Resolution test of the detonation conditions of the merger of two white dwarfs of $0.90 M_{\odot}$ and $0.89 M_{\odot}$ with an initial period of $25 \mathrm{~s}$. The left panel shows a simulation with $2 \times 10^{6}$ particles, the simulation shown in the right panel contains $10^{7}$ particles. Both panels show a density slice after the system has evolved for $32 \mathrm{~s}$. The black stars mark all particles with temperatures above $2 \times 10^{9} \mathrm{~K}$. expands. Temperatures exceeding $2 \times 10^{9} \mathrm{~K}$ are reached, but only for a few particles. These particles are the result of the additional energy release by thermonuclear combustion, because they ignite carbon that is subsequently burned. In simulations using identical initial conditions but no nuclear network, they do not exist. Some of them are also in dense enough regions to fulfill the criterion we require to form a detonation.

As the overall number of these particles is quite small, their exact properties have to be taken with caution. Therefore we have to check whether they show a barely resolved physical effect or are just a numerical artifact. This can be tested by redoing the simulation with significantly higher resolution.

The binary is not completely merged at the time the first hot spots form. Therefore, if a detonation occurs, it stops any further evolution of the merger and the merged object never reaches the final state of a white dwarf surrounded by a hot envelope found in previous simulations (e.g. Guerrero et al. 2004; Yoon et al. 2007; Lorén-Aguilar et al. 2009).

\subsection{Convergence study}

For a convergence study, we chose a slightly different binary with masses of $0.90 M_{\odot}$ and $0.89 M_{\odot}$ for the two WDs, respectively. As this binary has a mass ratio much closer to one, it was expected to merge more violently and therefore it should be easier to find extreme conditions in it.

Figure 3 shows the density of the two simulations of such a merger with $2 \times 10^{6}$ and $10^{7}$ particles, respectively. It demonstrates excellent agreement in the density structure between both simulations. In both simulations the particles with temperatures exceeding $2 \times 10^{9} \mathrm{~K}$ are found at the same locations. The number of these particles, however, increases from 11 to 127 from the lower resolved to the higher resolved simulation. As the number of particles only increases by a factor of five, the total mass at temperatures above $2 \times 10^{9} \mathrm{~K}$ is approximately twice as large in the high-resolution simulation. Therefore we can conclude that these hot particles are real, rather than a numerical artifact. The high-resolution simulation indicates that we underestimate the conditions in these hot-spots owing a lack of resolution in our standard setup.

\subsection{Additional effects on the detonation conditions}

There are two additional effects that may influence the formation of a detonation, but are basically ignored in our simulations. As discussed above, we have not resolved shocks at the interface very well. This effect can only be overcome by choosing a scheme that allows for refinement according to arbitrary criteria. Second, according to stellar evolution calculations there is a small helium shell on top of a carbon-oxygen white dwarf. For an isolated white dwarf around $0.9 M_{\odot}$, this shell is expected to contain $\approx 10^{-3}$ to $10^{-2} M_{\odot}$ of helium (Iben \& Tutukov 1985). It is known from the latest studies by Seitenzahl et al. (2009) that already a low-mass fraction of helium mixed into carbon fuel significantly lowers the density required to form a detonation. As the expected helium layer is located exactly in the region where the merger is most violent and the hotspots form, it may facilitate a detonation at even lower densities in our scenario.

In summary, there are reasons to believe that detonations may form for even a wider range of conditions than the ones we have assumed here.

\section{The impact of the mass ratio on the merger}

The violent merger scenario can only be realized frequently enough to account for a significant fraction of all type Ia supernovae if it also works for moderate mass differences between the two white dwarfs. Thus, we have to check how changing the mass ratio for binary systems with a fixed primary white dwarf mass affects the conditions during the merger. Only if mergers with a reasonable range also lead to forming a detonation, will the scenario be more than an exotic possibility. To test this, we compared four different mergers. All have the same primary mass of $0.9 M_{\odot}$, but different secondary masses of $0.89 M_{\odot}$, $0.81 M_{\odot}, 0.76 M_{\odot}$, and $0.7 M_{\odot}$, which give mass ratios of 0.99 , $0.9,0.84$, and 0.78 . In our simulations, they are set up with initial periods of $25 \mathrm{~s}, 33 \mathrm{~s}, 36 \mathrm{~s}$, and $40 \mathrm{~s}$, respectively.

Figure 4 shows the densities and temperatures of all particles of these simulations at the time the conditions are most favorable for a detonation, as well as temperature slices through the centers of the binaries. Obviously, there are considerable differences between these four systems. First, with decreasing mass ratio, the merger becomes less violent. While the mergers with mass ratios of $0.99,0.9$, and 0.84 produce several hot particles that ignite carbon and reach temperatures above $2 \times 10^{9} \mathrm{~K}$, this is not the 

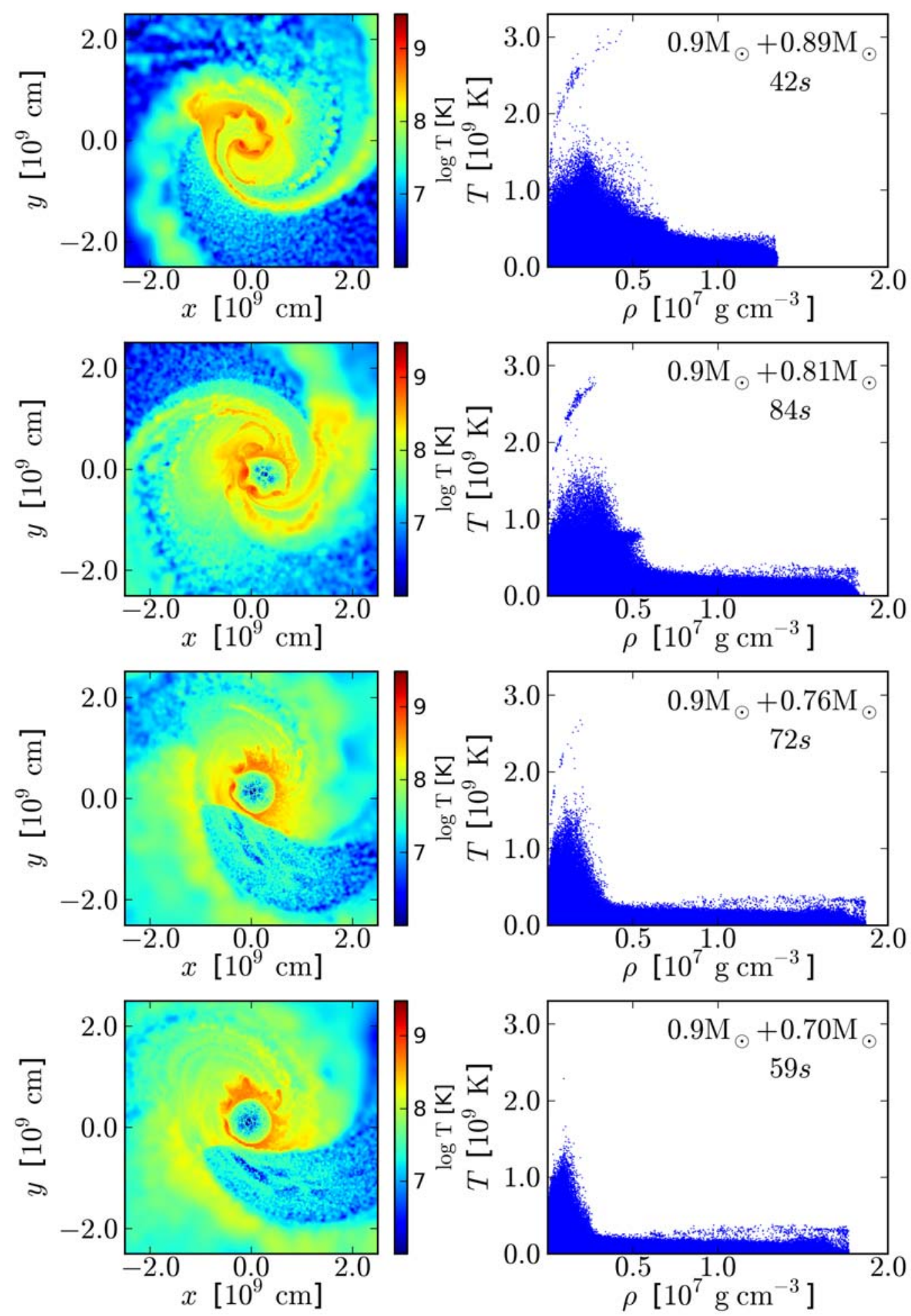

Fig. 4. Temperature slice and density-temperature scatter diagram for four mergers with the same primary white dwarf, but different secondary white dwarfs, that have mass ratios of $0.99,0.9,0.84$, and 0.78 . The rows show a temperature slice (on the left) and the distribution of all particles in temperature-density space (on the right) approximately at the time when there are the most favorable conditions for a detonation. Red/blue colors indicate high/low temperatures.

case for the merger with the lowest mass ratio. It is still possible that resolving the interaction region better will also show hotter particles; however, at the moment it seems more likely that the merger is just not violent enough below a certain mass ratio to ignite a detonation. This suggests a limiting mass ratio for the violent merger scenario of around 0.8 .
Another difference is the dynamical effect of the merger on the primary white dwarf. In a nearly equal mass merger, the primary star is heavily distorted, very similar to the equal mass merger described in Pakmor et al. (2010). For a mass ratio of 0.9 , the primary white dwarf remains unaffected in the center, but its surface is distorted. For the lower mass ratios, it stays 
completely intact and cool and is surrounded by the material of the disrupted less massive companion.

The effect of the merger on the primary white dwarf can also be seen in the right panels of Fig. 4 . In the $q=0.99$ merger, the central density of the remaining white dwarf is lower than in the other cases. As for these mergers the amount of ${ }^{56} \mathrm{Ni}$ produced is sensitive to the central density of the remaining white dwarf, this leads to lower ${ }^{56} \mathrm{Ni}$ masses in mergers with higher mass ratios.

While this does not change the scenario fundamentally, it breaks the relation between mass of the primary white dwarf and the final ${ }^{56} \mathrm{Ni}$ mass of the explosion for dim explosions. For explosions of mergers of more massive white dwarfs, however, where most of the ${ }^{56} \mathrm{Ni}$ is produced in nuclear statistical equilibrium, this will only be a minor effect. As shown in Fig. 4, with decreasing mass ratio the merger becomes less violent, and the density in the hotspots drops. This may make the formation of a detonation more difficult for lower mass ratios. For the lowest mass ratio no violent merger occurs and we see the onset of a different merger regime in which an accretion disk forms around primary white dwarf.

\section{Comparison with observations}

\subsection{Composition of the ejecta}

Figure 5 shows the ejecta composition of the thermonuclear explosion of the merger of two $0.89 M_{\odot}$ as described in Pakmor et al. (2010), which is close to the merger with a mass ratio of 0.99 described above. The final composition contains $0.03 M_{\odot}$ of carbon, $0.54 M_{\odot}$ of oxygen, $1.05 M_{\odot}$ of intermediate-mass elements, and $0.1 M_{\odot}$ of iron group elements. As the densities at the time the explosion happens do not exceed $2 \times 10^{7} \mathrm{~g} \mathrm{~cm}^{-3}$, nuclear burning does not reach nuclear statistical equilibrium any where in the object. Instead, iron group elements are only produced by incomplete silicon burning.

As the nuclear burning in the detonation takes place at low densities, electron captures are not efficient. Therefore the initial electron fraction $Y_{\mathrm{e}}$ of the unburned material is conserved throughout the nuclear burning. When of $Y_{\mathrm{e}}=0.5$, the iron group elements consist of almost pure ${ }^{56} \mathrm{Ni}$. A lower initial $Y_{\mathrm{e}}$ is equivalent to more neutron-rich material in the preexplosion composition (i.e. more ${ }^{22} \mathrm{Ne}$ resulting from the $\mathrm{He}$ burning phase). It leads to the production of some amount of stable iron replacing some of the ${ }^{56} \mathrm{Ni}$. In total, however, the fraction of stable iron group elements produced is very small.

As shown in Fig. 5, iron group elements are only located in the center, surrounded by intermediate-mass elements that are mixed with oxygen. Most carbon is found in the outermost parts, but there is some mixing with intermediate-mass elements. It is important to note that both carbon and oxygen are at different locations than the iron group elements; i.e., there is no mixing of unburned material with iron group elements. There is also a clear difference between the distribution in the $x$ - $y$-plane, which is the plane of rotation and the distribution along the $z$-axis. Along the $z$-axis, the iron group elements are spread out much more, which means that there are iron group elements at higher velocities in this direction than within the $x$-y-plane. The opposite trend is observed for oxygen and intermediate-mass elements, which reach farther out in the plane of rotation than perpendicular to it. This is a result of the density structure of the merged object through which the detonation propagates. Consequently, lightcurves and spectra of this explosion are expected to show considerable viewing angle dependence (for viewing angle dependent lightcurves see Pakmor et al. 2010).

\subsection{Comparison with other models and observations}

Compared to the standard W7 explosion model of Nomoto et al. (1984), our explosion models contain about $25 \%$ more mass, but the kinetic energy of the ejecta is about the same, so the velocities are lower on average. Figure 6 shows the mass distribution in velocity space in comparison to the W7 model. The merger has more mass at velocities below $12000 \mathrm{~km} \mathrm{~s}^{-1}$ and significantly less mass at higher velocities. With a ${ }^{56} \mathrm{Ni}$ yield of only around $0.1 M_{\odot}$, it is in the range of subluminous SNe Ia. We argue that it falls into the class of 1991bg-like supernovae described by e.g. Leibundgut et al. (1993) and Taubenberger et al. (2008). For one 1991bg-like supernova, SN 2005bl, a detailed spectral analysis is presented by Hachinger et al. (2009). In this study the authors find that decent models for the observed spectra require significantly less mass at higher velocities than what is inferred from the W7 mass profile. This agrees with the results of our simulations. Figure 6 shows a comparison between our final abundance pattern and the results of the abundance tomography for SN 2005bl. Only a qualitative match can be expected here, as the exact abundances depend on the density profiles, where Hachinger et al. (2009) used somewhat different assumptions. Despite differences in details, the main features are in qualitative agreement. There are basically three layers that differ in composition. The outermost layers are dominated by oxygen with contributions of unburned carbon and some amount of intermediatemass elements. Below about $11000 \mathrm{~km} \mathrm{~s}^{-1}$ intermediate-mass elements dominate down to very low velocities. Carbon is found down to $\approx 9000 \mathrm{~km} \mathrm{~s}^{-1}$. Iron group elements are present up to velocities of $\approx 7500 \mathrm{~km} \mathrm{~s}^{-1}$. In our model they only dominate in the very innermost part of the ejecta that is not probed by the photospheric spectra used in Hachinger et al. (2009).

The composition pattern of the ejecta of SN 2005bl inferred from abundance tomography is suggestive of a detonation at low densities as provided by our model. In particular, that iron group elements co-exist with intermediate-mass elements down to very low velocities but - at least as far as the observations can probe it - never dominate would be hard to explain with mixing alone. It strongly points to an extended range of incomplete silicon burning.

The abundances from our simulations shown in Fig. 6 are angle-averaged. Taking the asymmetry of the explosion into account as shown in Fig. 5, there is obviously some angle dependence in the composition in velocity space. This asymmetry will also affect the polarization of the observed spectra. Even though no spectrapolarimetry data is available from SN 1991bg or SN 2005bl, there are polarization spectra from SN 1999by, which fall into the same class of objects. Howell et al. (2001) find that this supernova shows continuum polarization of $\approx 0.3 \%-0.8 \%$. This marks an important difference to normal SNe Ia, which do not show continuum polarization (Wang \& Wheeler 2008). Our model provides a natural explanation for the comparatively high degree of polarization in spectra of 1991bglike objects, since the explosion is intrinsically asymmetric.

As this model assumes a progenitor system that is most likely different from that of normal SNe Ia, it may also explain the observed trend of $1991 \mathrm{bg}$-like SNe to favoring early-type galaxies, hence old stellar populations (e.g. Howell 2001). A long inspiral time of the original WD-WD binary leads to a long delay time between the formation of the progenitor system and the supernova explosion in our model.

Overall, the violent merger model presented here is not only the first realistic hydrodynamical model of a thermonuclear explosion of a WD that is able to produce ${ }^{56} \mathrm{Ni}$ masses low 


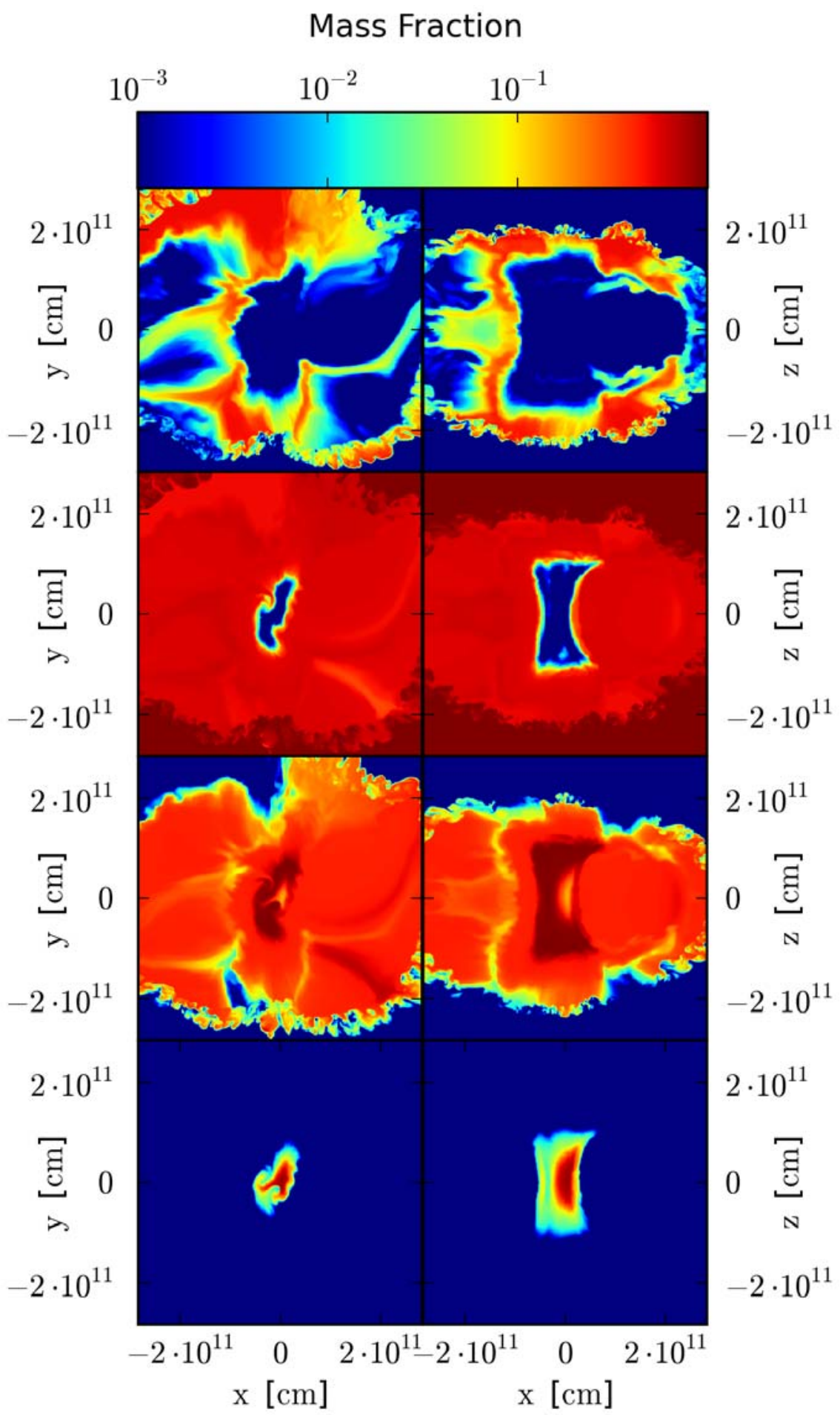

Fig. 5. Final composition of the ejecta of the the explosion of a merger of two $0.89 M_{\odot}$ white dwarfs. At this time, $100 \mathrm{~s}$ after the explosion, the ejecta expand homologously. The panels show from the top to the bottom the abundances of carbon, oxygen, intermediate-mass elements, and iron group elements. The left and right panels show the system viewed face-on and edge-on, respectively. enough to explain subluminous SN Ia, but it also shows remarkable agreement with observable features of $1991 \mathrm{bg}$-like objects in a more detailed comparison. A more quantitative comparison based on synthetic lightcurves and spectra can be found in Pakmor et al. (2010).

\section{Conclusion}

In this paper we investigate the effect of variations in the mass ratio of merging white dwarf binaries on the violent merger scenario proposed by Pakmor et al. (2010). We first concentrate on a prototypical realization of this scenario: the case of a double degenerate binary system with a total mass of $1.61 M_{\odot}$ and a mass ratio of 0.9. We follow the evolution of the binary system and subsequent merging event in smoothed particle hydrodynamical simulations.

We discuss in detail the conditions that arise in the violent merging event and find that it is reasonable to assume that they lead to the formation of a detonation. We also show that, while we are only barely resolving the most important part of the simulation (i.e. the interface between the two white dwarfs, where the detonation forms), a very high-resolution run comprising $10^{7}$ particles turns out to favor the formation of a detonation even more.

To estimate the influence on the mass ratio of the merging WDs on the detonation ignition conditions, we compared 


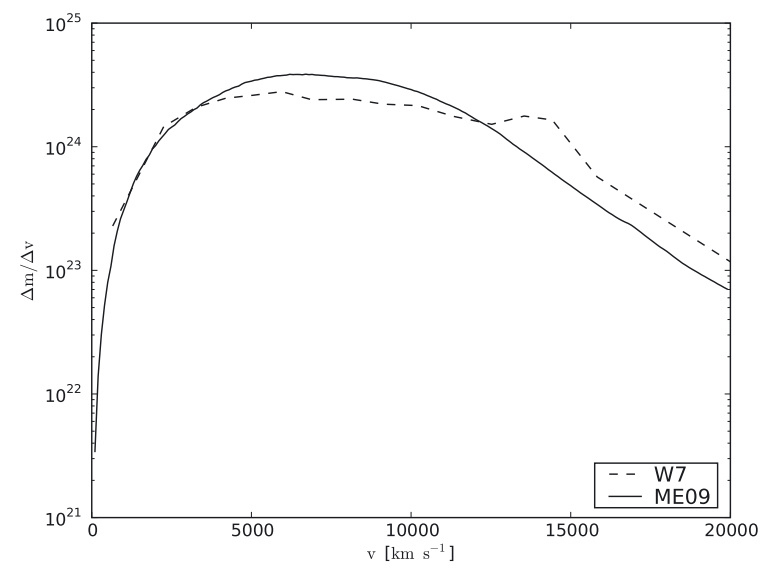

Fig. 6. Mass distribution in velocity space resulting from the thermonuclear explosion following a violent merger of two white dwarfs of $0.9 M_{\odot}$. The standard Chandrasekhar-mass SN Ia model W7 is overplotted for comparison.

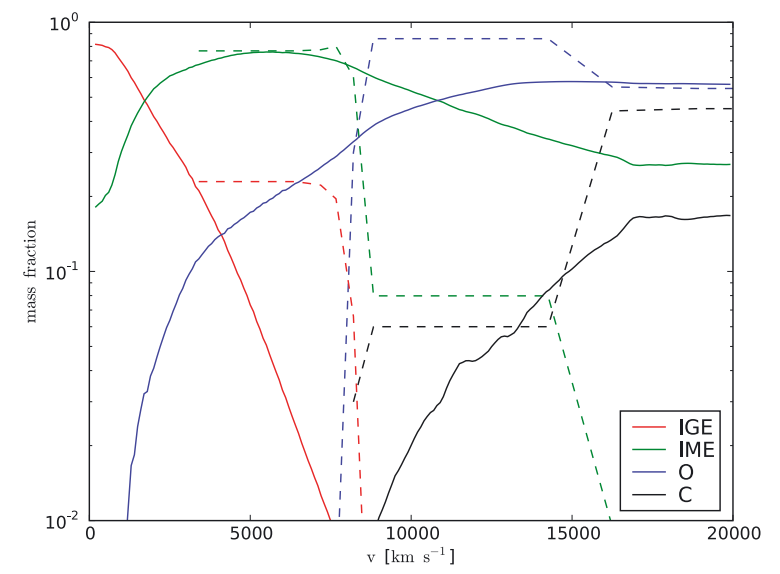

Fig. 7. Angle-averaged composition in velocity space (straight lines) as the result of the thermonuclear explosion following a violent merger of two white dwarfs of $0.9 M_{\odot}$. It is compared to the results of an abundance tomography study of SN 2005bl by Hachinger et al. (2009) (dashed lines).

mergers with a primary white dwarf mass of $0.9 M_{\odot}$, but different mass ratios ranging from 0.76 to unity. We find that merging systems with a mass ratio above $\sim 0.8$ detonate, but those with a mass ratio below 0.8 fail to do so.

Finally, we compared the final abundance structure in the ejecta of a thermonuclear explosion after the violent merger of two white dwarfs with $\sim 0.9 M_{\odot}$ with observed properties of faint SN 1991bg-like supernovae. The ejecta have higher mass, but lower velocities than the standard Chandrasekhar-mass W7 model. The abundance distribution of the model provides a good qualitative match with the composition found from the abundance tomography of SN 2005bl by Hachinger et al. (2009). Moreover, the model could provide a natural explanation for the polarization found in SN 1999by and connect the preference of SN 1991bg-like objects to occur in old stellar populations with their progenitors.

Of course, future studies are needed to explore the parameter space of different white dwarf masses and mass ratios in the violent merger scenario. Only this will allow us to understand the complete range of possible outcomes and the contribution of this channel to the observed sample of SNe Ia.

Acknowledgements. This work was supported by the Deutsche Forschungsgemeinschaft via the Transregional Collaborative Research Center TRR 33 "The Dark Universe", the Excellence Cluster EXC153 "Origin and Structure of the Universe" and the Emmy Noether Program (RO 3676/1-1). The simulations were carried out at the Computer Center of the Max Planck Society, Garching, Germany and the John von Neumann Institute for Computing (NIC) in Jülich, Germany (project HMU14).

\section{References}

Benz, W., Cameron, A. G. W., Press, W. H., \& Bowers, R. L. 1990, ApJ, 348, 647

Body, K. M. 1997, PhD Thesis, Department of Mechanical Engineering, McGill University, Montreal, Quebec, Canada

Branch, D., Fisher, A., \& Nugent, P. 1993, AJ, 106, 2383

Bravo, E., \& García-Senz, D. 2006, ApJ, 642, L157

Dan, M., Rosswog, S., \& Brüggen, M. 2009, J. Phys. Conf. Ser., 172, 012034

D’Souza, M. C. R., Motl, P. M., Tohline, J. E., \& Frank, J. 2006, ApJ, 643, 381

Filippenko, A. V., Richmond, M. W., Matheson, T., et al. 1992, ApJ, 384, L15

Fink, M., Hillebrandt, W., \& Röpke, F. K. 2007, A\&A, 476, 1133

Fink, M., Röpke, F. K., Hillebrandt, W., et al. 2010, A\&A, 514, A53

Guerrero, J., García-Berro, E., \& Isern, J. 2004, A\&A, 413, 257

Guillochon, J., Dan, M., Ramirez-Ruiz, E., \& Rosswog, S. 2010, ApJ, 709, L64

Hachinger, S., Mazzali, P. A., Taubenberger, S., Pakmor, R., \& Hillebrandt, W. 2009, MNRAS, 399, 1238

Hillebrandt, W., \& Niemeyer, J. C. 2000, ARA\&A, 38, 191

Howell, D. A. 2001, ApJ, 554, L193

Howell, D. A., Höflich, P., Wang, L., \& Wheeler, J. C. 2001, ApJ, 556, 302

Iben, Jr., I., \& Tutukov, A. V. 1984, ApJS, 54, 335

Iben, Jr., I., \& Tutukov, A. V. 1985, ApJS, 58, 661

Jordan, IV, G. C., Fisher, R. T., Townsley, D. M., et al. 2008, ApJ, 681, 1448

Kasen, D., Röpke, F. K., \& Woosley, S. E. 2009, Nature, 460, 869

Khokhlov, A. M., Oran, E. S., \& Wheeler, J. C. 1997, ApJ, 478, 678

Leibundgut, B., Kirshner, R. P., Phillips, M. M., et al. 1993, AJ, 105, 301

Li, W., Leaman, J., Chornock, R., et al. 2010, MNRAS, accepted [arXiv: 1006.4612]

Lorén-Aguilar, P., Isern, J., \& García-Berro, E. 2009, AIP Conf. Ser., 1122, 320

Mazzali, P. A., Nomoto, K., Cappellaro, E., et al. 2001, ApJ, 547, 988

Meakin, C. A., Seitenzahl, I., Townsley, D., et al. 2009, ApJ, 693, 1188

Motl, P. M., Frank, J., Tohline, J. E., \& D’Souza, M. C. R. 2007, ApJ, 670, 1314

Nomoto, K., \& Iben, Jr., I. 1985, ApJ, 297, 531

Nomoto, K., \& Kondo, Y. 1991, ApJ, 367, L19

Nomoto, K., Thielemann, F.-K., \& Yokoi, K. 1984, ApJ, 286, 644

Pakmor, R., Röpke, F. K., Weiss, A., \& Hillebrandt, W. 2008, A\&A, 489, 943

Pakmor, R., Kromer, M., Röpke, F. K., et al. 2010, Nature, 463, 61

Phillips, M. M. 1993, ApJ, 413, L105

Rauscher, T., \& Thielemann, F.-K. 2000, Atom. Data Nucl. Data Tables, 75, 1

Röpke, F. K., \& Niemeyer, J. C. 2007, A\&A, 464, 683

Röpke, F. K., Hillebrandt, W., Schmidt, W., et al. 2007a, ApJ, 668, 1132

Röpke, F. K., Woosley, S. E., \& Hillebrandt, W. 2007b, ApJ, 660, 1344

Ruiter, A. J., Belczynski, K., \& Fryer, C. 2009, ApJ, 699, 2026

Saio, H., \& Nomoto, K. 1998, ApJ, 500, 388

Scalzo, R. A., Aldering, G., Antilogus, P., et al. 2010, ApJ, 713, 1073

Seitenzahl, I. R., Meakin, C. A., Townsley, D. M., Lamb, D. Q., \& Truran, J. W. 2009, ApJ, 696, 515

Sim, S. A., Röpke, F. K., Hillebrandt, W., et al. 2010, ApJ, 714, L52

Springel, V. 2005, MNRAS, 364, 1105

Taubenberger, S., Hachinger, S., Pignata, G., et al. 2008, MNRAS, 385, 75

Timmes, F. X., \& Arnett, D. 1999, ApJS, 125, 277

Wang, L., \& Wheeler, J. C. 2008, ARA\&A, 46, 433

Webbink, R. F. 1984, ApJ, 277, 355

Whelan, J., \& Iben, I. J. 1973, ApJ, 186, 1007

Yoon, S.-C., Podsiadlowski, P., \& Rosswog, S. 2007, MNRAS, 380, 933

Zel'dovich, Y. B., Librovich, V. B., Makhviladze, G. M., \& Sivashinskii, G. I. 1970, J. Appl. Mech. Tech. Phys., 11, 264 